\title{
Morphological and Mechanical Properties of Poly(ethylene terephthalate) Gel and Melt Films in Terms of the Crystal Lattice Modulus, Molecular Orientation, and Small Angle X-Ray Scattering Intensity Distribution
}

\author{
Masaru Matsuo* and Chie SAwataRi ${ }^{\dagger}$ \\ Department of Clothing Science, Faculty of Home Economics, \\ Nara Women's University, Nara 630, Japan
}

(Received October 19, 1989)

\begin{abstract}
Deformation mechanism of polyethylene terephthalate (PET) gel and melt films was investigated in terms of morphological aspects by scanning electron microscopy, small angle $\mathrm{X}$-ray scattering, and wide angle X-ray difiraction. The morphological properties were discussed in relation to the temperature dependence of the dynamic complex modulus. The storage modulus of the melt film with a draw ratio of 20 was $19.5 \mathrm{GPa}$ at $20^{\circ} \mathrm{C}$, while that of the gel film with a draw ratio of 7 was $16.2 \mathrm{GPa}$. The apparent crystal lattice modulus of the (105) plane was measured by X-ray diffraction. Using the apparent value, the real value of the crystal lattice modulus was estimated to be $118 \mathrm{GPa}$ through a somewhat complicated geometrical treatment. The storage moduli for both the specimens are much lower than the crystal lattice modulus. In order to study the large difference, the crystallite orientation was estimated in terms of the second order orientation factor of the $c$-axis and the theoretical analysis of small angle X-ray scattering patterns. It turns out that under oriented crystallization, the preferential orientation of the $c$-axis is attributed to the rotation of crystallites with respect to the stretching direction but not to a crystal transformation from a folded to a fibrous type. Such a orientation mode is thought to hamper ultradrawing to produce high modulus PET films.
\end{abstract}

KEY WORDS Polyethylene Terepthalate / Gel Film / Melt Film / Crystal Lattice Modulus / Oriented Crystallization /

Since 1972, the preparation of polymeric fibers and films with high modulus has been extensively investigated and results of interest have been obtained for polyethylene ${ }^{1-8}$ and polypropylene. $^{8,10}$ Recently, Matsuo et al. produced ultradrawn polyethylene and polypropylene films whose Young's moduli at $20^{\circ} \mathrm{C}$ are $216 \mathrm{GPa}^{11}$ and $40.4 \mathrm{GPa},{ }^{12,13}$ respectively. They are nearly equal to their crystal lattice moduli measured by X-ray diffraction. High modulus fibers and films, however, have so far only realized for poly- $\alpha$-olefine polymers by the ultra-drawing of dried gel films. Unfortunately, the application range of poly- $\alpha$-olefine fibers is limited by the flexibility of the individual modulus and the low melting point. Therefore, it is important to achieve ultradrawing of other crystalline polymers such as polyesters with melting points above $200^{\circ} \mathrm{C}$ and with high molecular rigidity.

Among polyesters, poly(ethylene terephthalate) (PET) has been extensively studied in terms of morphological and mechanical properties because of outstanding commercial utility of this polymer. Most of studies have been carried out using compression-molded sheets as well as as-spun fibers with wide variations in crystallinity. ${ }^{14-22}$ However, there

* To whom all correspondence should be addressed.

† Present address: Faculty of Education, Shizuoka University, Shizuoka 422, Japan. 
has been no report to pursue the improvement of the mechanical properties of fibers (or films) which are prepared by gelation/crystallization from solutions, according to the method of Smith and Lemstra. ${ }^{6,7}$ This is probably due to the difficulty in preparing PET with ultra-high molecular weight, since PET is obtained by a polycondensation. Indeed, the experimental results in the previous work indicate that several kinds of polyethylene with molecular weights $<3 \times 10^{5}$ could not form a film by gelation/crystallization from solutions in the concentration range $0.1-5 \% .^{23}$

Efforts have been made to develop the technique for preparing PET gel films with high drawability, using PET pellets with highest molecular weight $\left(1.5 \times 10^{5}\right)$ available at present. The resultant gel films are stretched in a hot oven. The mechanical properties and the crystallite orientation were discussed in comparison with those of drawn melt films. Furthermore, the Young's moduli of the drawn films are discussed in relation to the crystal lattice modulus estimated from X-ray difiraction.

\section{EXPERIMENTAL}

Crude pellets of PET with molecular weight of $1.5 \times 10^{5}$ were treated with ethanol for $30 \mathrm{~h}$ in a Soxhlet's extractor before their use as specimens. Three kinds of solvents, nitrobenzene, $m$-cresol, and dichloroacetic acid were used. Three kinds of solutions, containing $5 \%$ of PEt and $0.1 \%$ of anti-oxidant di- $t$-butyl- $p$-cresol in solvents were prepared by heating well-blended polymer/solventmixtures at $190^{\circ} \mathrm{C}$ for $30-50 \mathrm{~min}$ under nitrogen. Each hot homogenized solution was poured into an aluminum tray that was surrounded by ice water to form a gel. The solvent was allowed to evaporate from the gels at ambient conditions. The nearly-dried gels were vacuumdried to remove residual traces of the solvent.

The extracted pellets were sandwiched between Teflon sheets at $190^{\circ} \mathrm{C}$ for $30 \mathrm{~min}$ at a pressure of $14 \mathrm{MPa}$. The molten samples were quenched by being plunged into an ice-water bath to prepare amorphous specimens.

The densities of the specimens were measured by pycnometer in a mixture of $n$-heptane and carbon tetrachloride as a medium. The degree of crystallinity was calculated by assuming the densities of the crystal and amorphous regions to be 1.455 and $1.335 \mathrm{~g}$ $\mathrm{cm}^{-3}$, respectively. ${ }^{24}$ The thermal behavior was estimated in terms of melting endotherms of differential calorimatry (DCS) curves. Dried gels, weighting $5 \mathrm{mg}$, were placed in a standard aluminum sample pan. Films were heated at a constant rate of $10^{\circ} \mathrm{C} \mathrm{min}{ }^{-1}$.

Scanning electron micrographs were obtained with JSM-T300.

The complex dynamic tensile modulus was measured at a frequency of $10 \mathrm{~Hz}$ using a viscoelastic spectrometer (VES-f) obtained from Iwamoto Machine Co., Ltd. The length of the specimen between the jaws was about $40 \mathrm{~mm}$ and the width was about $1.5 \mathrm{~mm}$. During measurements, the films were subjected to a static tensile strain in order to place the sample in tension during the axial sinuosidal oscillation which had a peak deformation of $0.25 \%$. The complex dynamic modulus was measured by imposing a small dynamic strain to ensure linear viscoelastic behavior of the specimen. Before the measurements were made, all the specimens were annealed for $1 \mathrm{~h}$ at $230^{\circ} \mathrm{C}$ and cooled slowly to room temperature at a fixed dimension in the stretching direction.

The crystal lattice modulus of PET cannot be observed directly by the X-ray diffraction, since there exists no detectable crystal plane whose reciprocal lattice vector is parallel to the crystal chain axis. In the unit cell of PET, the reciprocal lattice vector of the (105) plane, among all the crystal planes, most closely parallels the $c$-axis. The $\mathrm{X}$-ray diffraction intensity was measured with a sintillation counter and a $12 \mathrm{~kW}$ rotating-anode X-ray generator (Rigaku RDA-rA operated at $200 \mathrm{~mA}$ and $40 \mathrm{kV}$ ). The X-ray beam was 
monochromatized with a curved graphite monochromatar. Crystal strain was provided by a constant-stretching apparatus which has been described elesewhere. ${ }^{11,13}$ The intensity distribution for the (105) plane was measured with point focusing using a system in which the incident beam was collimated by a circular collimator $2 \mathrm{~mm}$ in diameter, and the diffraction beam was detected by a square slit with dimensions $0.9 \times 0.9 \mathrm{~mm}$. The specimen was mounted horizontally in the stretching clamps of the apparatus in such a way that the tilting angle between the film normal direction and the incident beam was $9.5-10^{\circ}$. The intensity distribution was measured with a setepscanning device at a step interval of $0.1^{\circ}$, each at a fixed time of $100 \mathrm{~s}$, in the range $40.5-45^{\circ}$ (twice the Bragg angle).

Small angle X-ray scattering (SAXS) patterns were obtained with a flat film camera. The exposure time for all the specimens was $100 \mathrm{~h}$ both in the case of through and end views.

\section{RESULTS AND DISCUSSION}

Figure 1 shows the change in the appearance of the dry gels prepared from three kinds of solvents under scanning electron microscopy. The textures prepared from nitrobenzene and $m$-cresol is fibrillar tissue, while that from dichloroacetic acid is spherulitic one indicating the existence of polymer spherulites. The texture from nitrobenzene seems to be composed of fibrillar tissue comprising interconnected lamellar crystals and that from $m$-cresol becomes indistinct fibrillar tissue showing smooth surface. In a macroscopic sense, these dry gel did not form a film and they were composed of powder like single crystal mats. They were cracked on the drying at ambient conditions. This phenomenon is attributed to the fact that a number of coupling entanglement mesh is important to form a gel film, but, unfortunately, even the molecular weight of $1.5 \times 10^{5}$, the value being the highest among PET available at present, is not sufficient to ensure a suitable level of the entanglement mesh, because of higher crystallinities than $85 \%$ of the resultant gels. This concept has been demonstrated from the morphological properties of polyethylene gels prepared with different kinds of molecular weights. ${ }^{23}$ According to the previous work, ${ }^{23}$ the crystallinities of dried polyethylene gels were independent of molecular weights for the gels prepared from solutions in the concentration range $0.1-5 \%$. The crystallinities of ultra-high molecular weight polyethylene (UHMWPE) gel with molecular weight of $6 \times 10^{6}$ and low molecular weight polyethylene (LMWPE) gels with molecular weights $<3 \times 10^{5}$ were in the range $80-85 \%$. Only

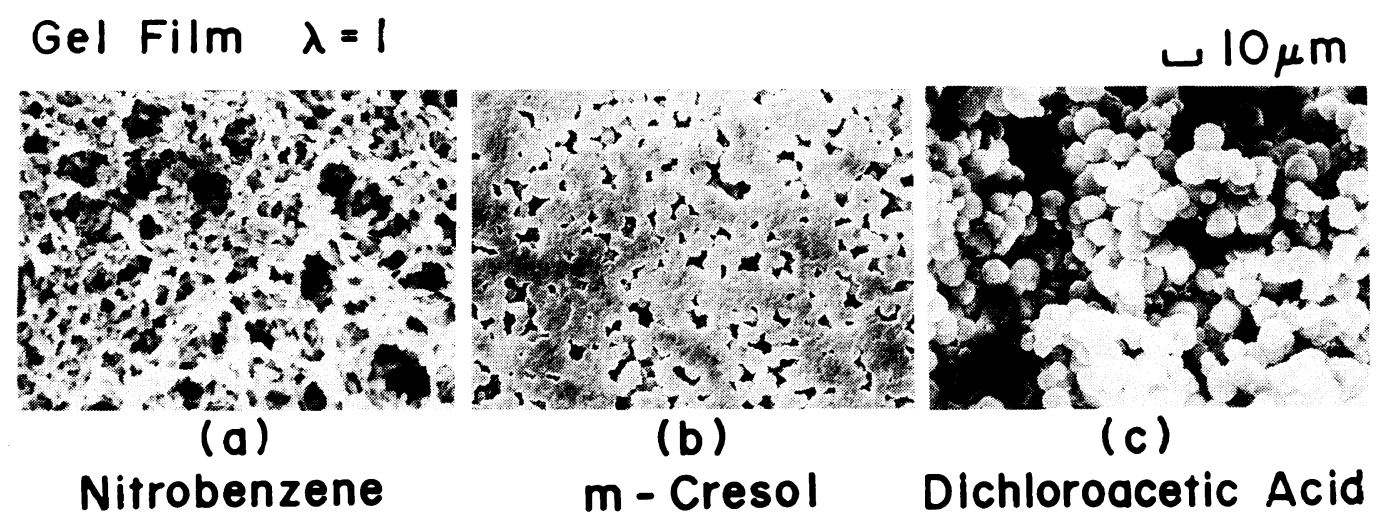

Figure 1. Scanning electron micrographs of dry gels of PET prepared from three kinds of solvents (a) nitrobenzene, (b) $m$-cresol, (c) dichloroacetic acid. 


\title{
Gel Film $\lambda=1$
}

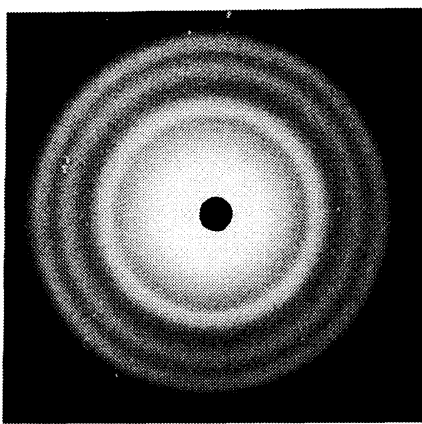

(a)

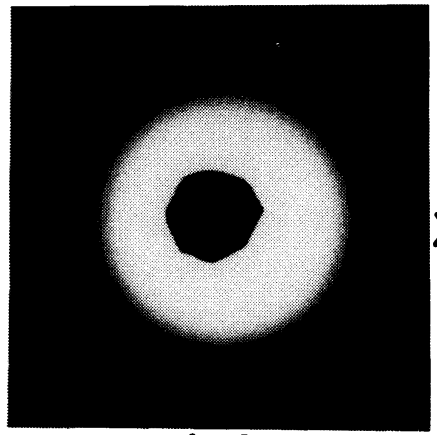

(b)

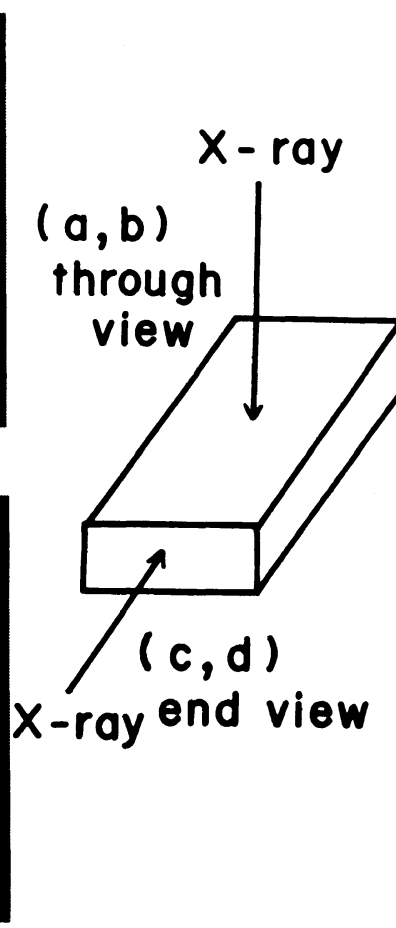

\begin{abstract}
(d)
ms of PET: WAXD (a) and SAXS (b) patterns (through view); WAXD
\end{abstract}
(c) and SAXS (d) patterns (end view).

UHMWPE gel could form a film. From this result, it was inferred that total amorphous chains per molecules for UHMWPE are sufficient enough to ensure a suitable level of the entanglement mesh to form a gel film, since the length of total segments to form amorphous phase in one polymer chain of UHMWPE is much higher than that of LMWPE. Judging from this concept, it would be expected that the same interpretation may be constructed for the PET gels.

Figure 2 shows the WAXD and SAXS patterns for the gel film prepared from nitrobenzene solution. The WAXD patterns (a) and (c) show diffraction ringes and the SAXS patterns (b) and (d) show circular diffuse scatterings. The four patterns indicate that the PET gel is composed of crystal lamellae with random orientation within the gel. This orientation mode is quite different from the structures of gel films of polyethylene ${ }^{23}$ and polypropylene $^{12}$ whose the constituent lamellar crystals were highly oriented with their large flat faces parallel to the film surface.

The brittle PET gels were sandwiched between Teflon sheets at $225^{\circ} \mathrm{C}$ for $5 \mathrm{~min}$ at a pressure of $14 \mathrm{MPa}$ in order to increase the number of coupling entanglement mesh owing to partial melt of the gels. The resultant specimen formed a gel with about $50 \%$ crystallinity, indicating a $41 \%$ decrease from the original gel. The specimen prepared by this method termed "PET gel film" in order to distinguish from a molten sample which termed "PET melt film." Incidentally, the following experimental results in this paper were reported only for the specimens prepared from nitrobenzene solution, since the gels prepared 


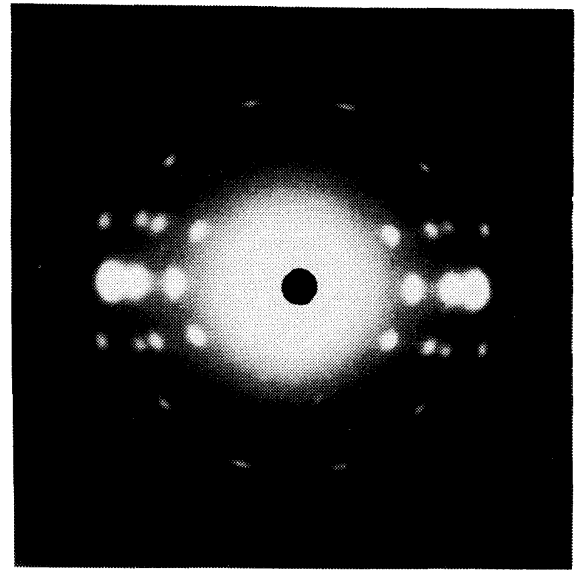

(a) Melt Press Film $\lambda=20$

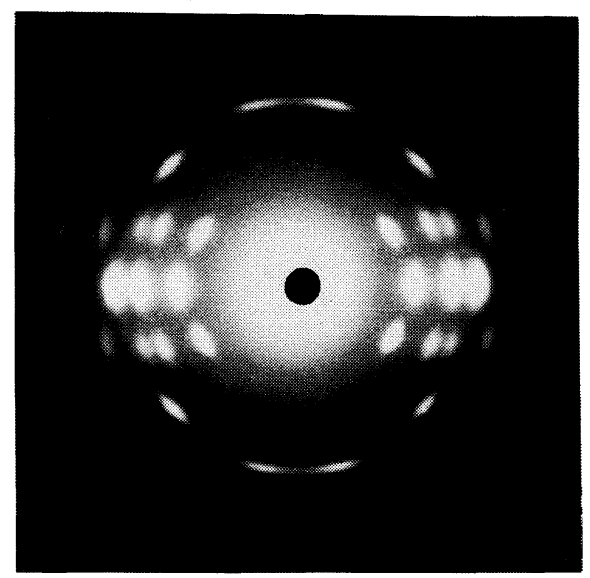

(b) Gel Film $\lambda=7$

Figure 3. WAXD patterns of drawn PET films (a) melt press film drawn to $\lambda=20$. (b) gel film drawn to $\lambda=7$.

from the other two solvents were, to all appearance, similar to that prepared from nitrobenzene.

The gel films were elongated to the maximum draw ratio of 7 in a hot oven at $250^{\circ} \mathrm{C}$ under nitrogen and the crystallinity of the drawn film was $58 \%$, while the melt films were also drawn to the maximum draw ratio of 20 . Elongation to the draw ratio of 20 was done in the second stage. The original specimen was first drawn to $\lambda=12$ in a hot water bath at $95^{\circ} \mathrm{C}$ and after vacuum-drying the specimen was drawn to 20 in a hot oven at $250^{\circ} \mathrm{C}$ under nitrogen in the second stage. The crystallinity of the resultant specimen was about $60 \%$.

Figure 3 shows the WAXD patterns (through view), in which the patterns in part (a) and (b) show the diffractions from the melt film $(\lambda=20)$ and the gel film $(\lambda=7)$, respectively. The diffraction spots in the pattern (a) indicate a high degree of crystallite orientation of the melt film with $\lambda=20$.

The extensive works of molecular orientation of PET was carried out by Ward et al., 25-27 in terms of the fourth order moment using the polarized fluorescence and nuclear magnetic resonance as well as in terms of the second order one using infra-red radiation and birefringence. On the basis of a series of experimental results, they found some interesting orientational behaviors of PET molecules such as planner orientation of benzene ring, preferential orientation of the polymer in the trans conformation, and rubber deformation of amorphous chain segments. In order to estimate the crystal orientation, more quantitatively using a method by Roe and Krigbaum, ${ }^{28,29}$ the orientation distribution functions of the reciprocal lattice vectors of the crystal planes were measured. Figures 4 and 5 show the orientation distribution functions, in which $q_{j}\left(\cos \theta_{j}\right)$ reveals the function of the reciprocal lattice vector of the $j$-th crystal plane. The functions indicate that the degree of crystallite orientation for the gel film is not as great as that for the melt film. Using the functions in Figures 4 and 5, the second order orientation factor $F_{20}^{j}$ of the $j$-th crystal plane may be given by the well known expression. 


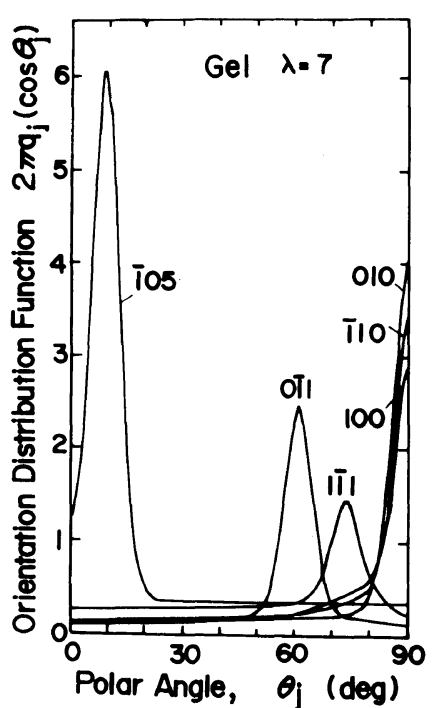

Figure 4. Oreintation distribution functions of the reciprocal lattice vectors of the six crystal planes of the gel films of PET with $\lambda=7$.

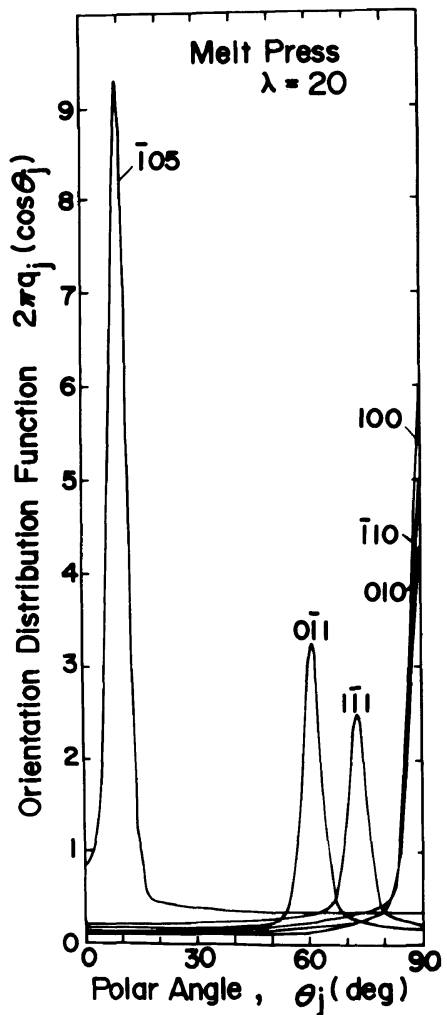

Figure 5. Orientation distribution functions of the reciporcal lattice vectors of the six crystal planes of the melt films of PET with $\lambda=20$.
$F_{20}^{j}=2 \pi \int_{0}^{\pi / 2} q_{j}\left(\cos \theta_{j}\right)\left(3 \cos ^{2} \theta_{j}-1\right) / 2 \sin \theta_{j} \mathrm{~d} \theta_{j}$

where $\theta_{j}$ is the angle between the $j$-th reciprocal lattice vector and the stretching direction. $F_{20}^{j}$ characterizes the crystallite orientation distribution, with variation between $-1 / 2$ and 1 . For random orientation of the $j$-th axis, $F_{20}^{j}$ is 0 , while for complete orientation parallel and perpendicular to the stretching direction, $F_{20}^{j}$ is unity and $-1 / 2$, respectively.

Now, using a procedure described by Krigbaum et al. ${ }^{28,29}$ one can obtain the second order orientation factor of crystallites as follows:

$$
\begin{aligned}
& F_{20}^{j}=F_{200} P_{2}\left(\cos \Phi_{j}\right) \\
& +(1 / 3)\left(F_{201} \cos \Phi_{j}-G_{201} \sin \Phi_{j}\right) P_{2}^{1}\left(\cos \Theta_{j}\right) \\
& +(1 / 12)\left(F_{202} \cos 2 \Phi_{j}-G_{202} \sin 2 \Phi_{j}\right) P_{2}^{2}\left(\cos \Phi_{j}\right)
\end{aligned}
$$

with

$$
\begin{aligned}
& P_{2}(x)=(1 / 2)\left(3 x^{2}-1\right) \\
& P_{2}^{1}(x)=3\left(1-x^{2}\right)^{1 / 2} x \\
& P_{2}^{2}(x)=3\left(1-x^{2}\right)
\end{aligned}
$$

In eq $2, \Theta_{j}$ and $\Phi_{j}$ are the polar and azimuthal angles of the reciprocal lattice vector $U_{j}$ with respect to the structural unit and they were given elsewhere. ${ }^{19}$ By measuring the orientation distribution functions of the reciprocal lattice vectors for each of the five crystal planes (100), (110), (010), (1111), and (011), the corresponding values of $F_{20}^{j}$ were calculated from eq 1 . Then, substituting the values of $F_{20}^{j}$ into eq 2 gives five sorts of simultaneous linear equations that can be solved to obtain the five unknown coefficients $F_{200}, F_{201}, F_{202}, G_{201}$, and $G_{202}$. Finally, with the knowledge of the values of these five coefficients, the orientation factors of the three principal crystallographic axes could be calculated.

Assuming that the birefringence effects of the amorphous phase, $\Delta_{a m}$, may be estimated by 
subtracting the crystalline contribution from the total birefringence, $\Delta_{t}$, we have

$$
\begin{gathered}
\Delta_{\mathrm{am}}=\frac{\Delta_{\mathrm{t}}-x_{\mathrm{c}} \Delta_{\mathrm{c}}}{1-x_{\mathrm{c}}} \\
\Delta_{\mathrm{am}}=\left\{n_{33}^{\mathrm{am}}-(1 / 2)\left(n_{11}^{\mathrm{am}}+n_{22}^{a m}\right)\right\} F_{200}^{\mathrm{am} \mathrm{m}^{\prime}}
\end{gathered}
$$

and

$$
\begin{aligned}
\Delta_{\mathrm{c}}= & \left\{n_{33}^{\mathrm{co}}-(1 / 2)\left(n_{11}^{\mathrm{co}}+n_{22}^{\mathrm{co}}\right)\right\} F_{200} \\
& +(1 / 4)\left(n_{11}^{\mathrm{co}}-n_{22}^{\mathrm{co}}\right) F_{202}
\end{aligned}
$$

where $X_{\mathrm{c}}$ is the degree of volume crystallinity. The parameters $n_{33}^{\text {co }}$ and $n_{11}^{\text {co }}$ are the refractive indexes along the $c$-axis and along the $a^{*}$ axis corresponding to the direction normal to the benzene ring. $n_{22}^{\text {co }}$ is the refractive index in the direction perpendicular to the $c$ and $a^{*}$ axes. $n_{33}^{\mathrm{am}}$ is the refractive index along the axis normal to the benzene ring and $n_{22}^{\mathrm{am}}$ and $n_{11}^{\mathrm{am}}$ are the refractive indexes perpendicular to the above axis. These values were calculated by Sakaguchi et $a l .{ }^{30}$ in the usual way by assuming the atomic arrangements within the crystal reported by de Daubeny et al. ${ }^{24}$ and the values of the bond polarizabilities according to Bunn and Daubeny ${ }^{31}$ and by neglecting the uncertain effect of the internal field within the crystal and of secondary bonds upon the principal polarizabilities. They gave

$$
\begin{aligned}
& n_{33}^{\mathrm{co}}=1.806 \\
& n_{22}^{\mathrm{co}}=1.733 \\
& n_{11}^{\mathrm{co}}=1.398 \\
& n_{33}^{\mathrm{am}}=1.362 \\
& n_{22}^{\mathrm{am}}=n_{11}^{\mathrm{am}}=1.687
\end{aligned}
$$

Table I. The second order orientation factors of the reciprocal lattice vectors of the six crystal plane, the principal crystallographic axes, and amorphous chain segments

\begin{tabular}{ccccccccccc}
\hline & $(100)$ & $(\overline{1} 10)$ & $(010)$ & $(1 \overline{1} 1)$ & $(0 \overline{1} 1)$ & $(\overline{1} 05)$ & $a$-Axis & $b$-Axis & $c$-Axis & Amorphous \\
\hline $\begin{array}{c}\text { Gel film } \\
(\lambda=7)\end{array}$ & -0.313 & -0.304 & -0.351 & -0.389 & -0.111 & 0.285 & -0.222 & -0.309 & 0.652 & - \\
\hline $\begin{array}{c}\text { Melt film } \\
(\lambda=20)\end{array}$ & -0.389 & -0.341 & -0.327 & -0.210 & -0.099 & 0.303 & -0.215 & -0.282 & 0.686 & 0.769 \\
\hline
\end{tabular}

Since, $n_{33}^{\mathrm{am}}<n_{22}^{\mathrm{am}}, F_{200}^{\mathrm{am}}$ corresponds to the second order orientation factor of the $a^{*}$ axis. In the case when the amorphous chain axes orient randomly around their own axes, the orientation factors of the other two axes perpendicular to the amorphous chain axis become equivalent with respect to the stretching direction. Then the orientation factor of the amorphous chain axes may be given as follows:

$$
F_{200}^{\mathrm{am}}=-2 F_{200}^{\mathrm{am} \prime}
$$

Table I presents values of $F_{20}^{j}$ and $F_{200}^{\text {am }}$. The value of $F_{200}^{\mathrm{am}}$ could not be estimated for the gel film, because of the difficulty in measuring the birefringence for whitening. Here it should be noted that the orientation of the $c$-axes is essentially different from that of the (105) plane which most closely parallels the $c$-axis among the reciprocal lattice vectors of the all crystal planes in the unit cell of PET. This means that the orientation factor of the $c$-axes should be calculated using the method described in eq 1 and 2 . The values of the $c$-axes for the melt and gel films indicate the preferential orientation of crystallites with respect to the stretching direction and the values of the $a$ and $b$ axes for both the specimens indicate the preferential orientation perpendicular to the stretching direction. Such a orientation mode exhibits a typical fibrous structure. The value of $F_{200}^{\mathrm{am}}$ is much more pronounced than that of $F_{200}$ of the $c$ axis for the drawn melt film. This indicates that under oriented crystallization, the preferential orientation of the $c$-axis is attributed to the rotation of crystallites by the tension of 


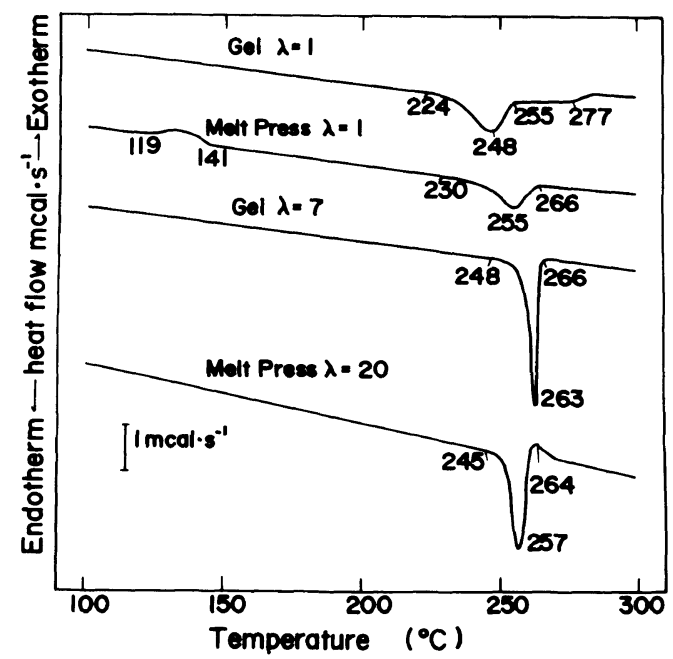

Figure 6. DSC curves of PET gel and melt films in undrawn and drawn states.

tie molecules that act as interlamellar crosslinks, which shall be discussed later in relation to SAXS patterns.

Figure 6 shows the changes in the profiles of DSC curves for the gel and melt films in an undeformed state and in their maximum draw ratios. The crystallizing exotherm appears around $135^{\circ} \mathrm{C}$ for the melt film because of the low crystallinity $(2 \%)$. The melting point and the shape of melting endotherm depend on the sample preparation conditions. The profile of the undrawn gel film shows a small peak similar to that of the melt film associated with thermal crystallization. Such a small peak cannot account for the high crystallinity above $85 \%$ of the undrawn gel film. Furthermore, it is noteworthy that the endotherms for the melt film drawn to $\lambda=20$ is less intense than that for the gel film drawn to $\lambda=7$ and the corresponding melting point is lower, although the crystallinity of the melt film $(60 \%)$ is a little bit higher than that of the gel film $(58 \%)$. The crystallinity of the undrawn gel film is higher than that of the drawn film. This phenomenon has never been observed for polyethylene ${ }^{8,11}$ and polypropylene ${ }^{12,13}$ The decrease in the crystallinity of poly(ethylene terephthalate) under elongation is probably due to a decrease in the paracrystalline content. This behavior, however, is not reflected in the DSC curves, although DSC measurements have the advantage of revealing small changes of crystal sizes, crystal defects, and crystal type that are impossible to detect on the basis of the density measured by pycnometry.

Figures 7(a) and (b) show the temperature dependences of the storage and loss moduli for the drawn gel film $(\lambda=7)$ and the drawn melt film $(\lambda=20)$, respectively, at a frequency of $10 \mathrm{~Hz}$. It can be seen that the storage modulus decreases with increasing temperature for both the films. This tendency is similar to the results which have been observed by a number of authors for semicrystalline polymers. The storage moduli of the gel and melt films at $20^{\circ} \mathrm{C}$ are about 16.2 and $19.5 \mathrm{GPa}$, respectively. These values are much lower than those of ultradrawn polyethylene and polypropylene films reported already. ${ }^{11,12}$

The loss moduli for both the specimens have two peaks around $110^{\circ} \mathrm{C}$ and at temperatures below $-50^{\circ} \mathrm{C}$, respectively. According to Yoshihara et al., ${ }^{13}$ the former peak corresponds to the summation of the $\alpha$ and $\beta$ transitions associated with the rotational relaxation of crystal grains within crystal lamellae and with orientational relaxation of amorphous chain segments, respectively. The latter peak was reported to correspond to the $\gamma$ transition associated with the relaxation concerning local deformation of amorphous chain segments. Anyway, the peak positions are independent of the sample preparation conditions.

Figure 8 shows a stress-strain relationship of the melt film drawn to $\lambda=20$. The original length of the specimen was about $40 \mathrm{~mm}$ and the cross-head speed was $2 \mathrm{~mm} \mathrm{~min}^{-1}$. The relationship shows a typical curve which has been well known for drawn polymeric materials. The Young's modulus and the tensile modulus at break were 16 and $0.48 \mathrm{GPa}$, respectively, at $30^{\circ} \mathrm{C}$. The Young's modulus is little bit lower than the storage modulus 


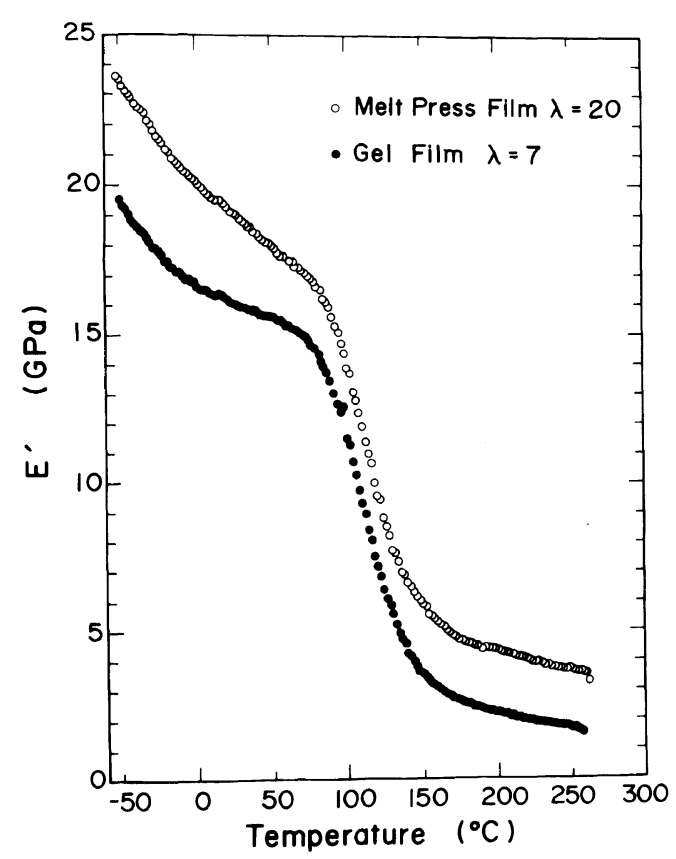

Figure 7(a). Temperature-dependence of the storage modulus of a drawn gel film $(\lambda=7)$ and a drawn melt film $(\lambda=20)$ at a frequency of $10 \mathrm{~Hz}$.

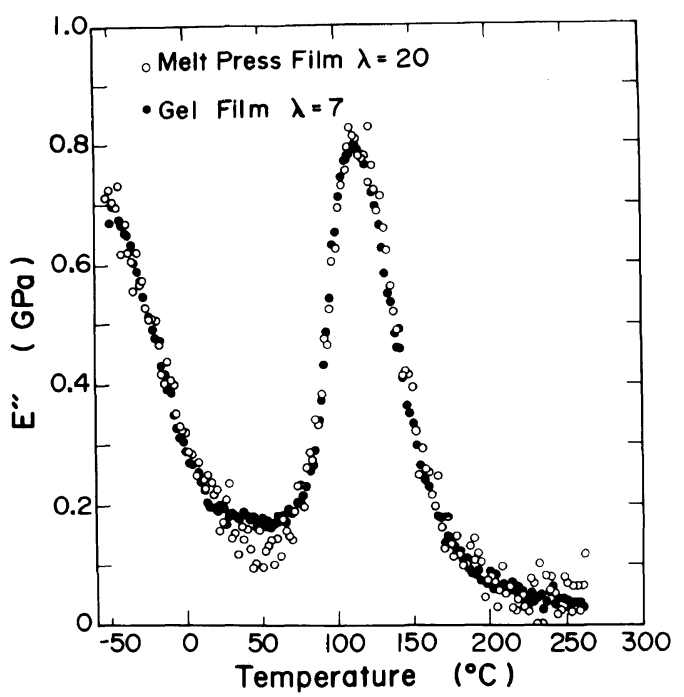

Figure 7(b). Temperature-dependence of the loss modulus of a drawn gel film $(\lambda=7)$ and a drawn melt film $(\lambda=20)$ at a frequency of $10 \mathrm{~Hz}$.

$(18.5 \mathrm{GPa})$ at $30^{\circ} \mathrm{C}$ shown in Figure $7(\mathrm{a})$, but the difference seems to be within experimental error.

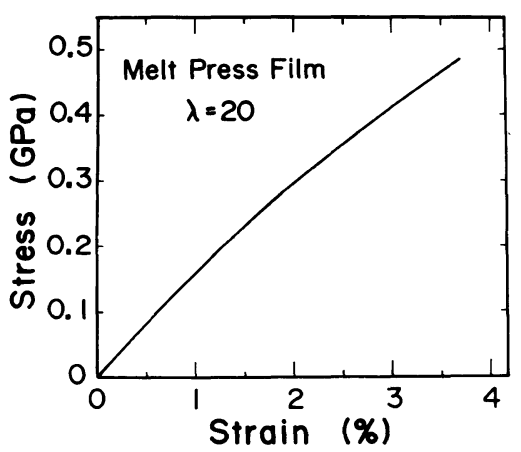

Figure 8. Stress-strain relationship of a drawn melt film $(\lambda=20)$ (strain rate $\left.5 \% \mathrm{~min}^{-1}\right)$.

Here it is of interest to measure the crystal lattice modulus in the direction of the $c$-axis (or fiber axis) in order to obtain the ultimate values of the Young's modulus of PET. The crystal lattice modulus was measured by Sakurada et al. ${ }^{32}$ using commercial PET fibers and the value was reported as $108 \mathrm{GPa}$. They substituted the apparent crystal lattice modulus of the (105) plane for the crystal lattice modulus in the direction of the $c$-axis, since the reciprocal lattice vector of the (105) plane, among all the crystal planes which can be detected by the X-ray diffraction, most closely parallels to the $c$-axis. As discussed before, however, the second order orientation factor of the $c$-axis is different from that of the (105) plane. The real value of the crystal lattice modulus in the direction of the $c$-axis is estimated through the rectification of the lattice modulus of the (105) plane. This treatment is essentially the same as that in the case of estimating the crystal lattice modulus or polypropylene. ${ }^{13}$

Figure 9 shows a schematic diagram defining the relationship between the reciprocal lattice vector of the (105) plane and the $c$-axis. $0-X_{1} X_{2} X_{3}$ is Cartesian coordinate within the specimen, with the $X_{3}$ axis lying along the stretching direction and $X_{2} X_{3}$ plane parallel to the surface of the specimen. The $U_{3}$ axis is taken in the direction of the reciprocal lattice vector of the (105) plane. The $U_{3}$ axis is defined 


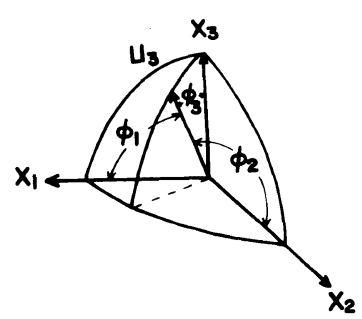

Figure 9. Schematic diagram showing the relationship between the $c$-axis direction ( $X_{3}$ axis) and the direction of the reciprocal lattice vector of the crystal (105) plane $\left(U_{3}\right.$ axis), in which Euler angles $\phi_{1}, \phi_{2}$, and $\phi_{3}$ specify the orientation of the $U_{3}$ axis with respect to the Cartesian coordinate $0-X_{1} X_{2} X_{3}$ fixed within a film specimen.

by polar angles $\phi_{3}, \phi_{2}$, and $\phi_{1}$, with respect to the $X_{3}, X_{2}$, and $X_{1}$ axes, respectively. In this system, if the $c$-axis (or fiber axis) orients perfectly in the stretching direction, it corresponds to the $X_{3}$ axis. This can be realized for ultradrawn films. However, the orientation of the $c$-axis has the distribution with respect to the $X_{3}$ axis as listed in Table I. Accordingly, this assumption cannot be constructed for the PET films used in this experiment. The rectification, however, is carried out using the above system in order to avoid the difficulties of mathematical procedure by the introduction of the orientational fluctuation effect.

The mechanical properties of a PET crystal unit showing a triclinic system can be described by Hook's law.

$$
\left(\begin{array}{l}
\varepsilon_{11} \\
\varepsilon_{22} \\
\varepsilon_{33} \\
\varepsilon_{23} \\
\varepsilon_{31} \\
\varepsilon_{12}
\end{array}\right)=\left(\begin{array}{llllll}
S_{11} & S_{12} & S_{13} & S_{14} & S_{15} & S_{16} \\
S_{21} & S_{22} & S_{23} & S_{24} & S_{25} & S_{26} \\
S_{31} & S_{32} & S_{33} & S_{34} & S_{35} & S_{36} \\
S_{41} & S_{42} & S_{43} & S_{44} & S_{45} & S_{46} \\
S_{51} & S_{52} & S_{53} & S_{54} & S_{55} & S_{56} \\
S_{61} & S_{62} & S_{63} & S_{64} & S_{65} & S_{66}
\end{array}\right)\left(\begin{array}{l}
\sigma_{11} \\
\sigma_{22} \\
\sigma_{33} \\
\sigma_{44} \\
\sigma_{55} \\
\sigma_{66}
\end{array}\right)
$$

relating strain $\varepsilon$ to stress $\sigma$, where $S_{i j}$ is the elastic compliance. The subscripts 3 and 2 are chosen in the directions of the $X_{3}$ and $X_{2}$ axes, respectively. When uniform stress $\sigma=\left(\sigma_{33}\right)$ is direcred along the $X_{3}$ axis as shown in Figure
9 , the components of strain $\varepsilon_{i j}$ in eq 9 may be given by

$$
\left.\begin{array}{l}
\varepsilon_{11}=S_{13} \sigma \\
\varepsilon_{22}=S_{23} \sigma \\
\varepsilon_{33}=S_{33} \sigma \\
\varepsilon_{23}=S_{43} \sigma \\
\varepsilon_{31}=S_{53} \sigma \\
\varepsilon_{12}=S_{63} \sigma
\end{array}\right\}
$$

According to the geometrical arrangement in Figure 9, the strain $\varepsilon_{33}^{\prime}$ in the direction of the $U_{3}$ axis may be given by

$$
\begin{aligned}
\varepsilon_{33}^{\prime}= & \cos ^{2} \phi_{3} \varepsilon_{33}+\cos ^{2} \phi_{2} \varepsilon_{22}+\cos ^{2} \phi_{1} \varepsilon_{11} \\
& +\cos \phi_{1} \cos \phi_{2} \varepsilon_{12}+\cos \phi_{1} \cos \phi_{3} \varepsilon_{13} \\
& +\cos \phi_{2} \cos \phi_{3} \varepsilon_{23}
\end{aligned}
$$

Since the uniaxially drawn specimen has fiber symmetry, the $U_{3}$ axis exhibits a random orientation with respect to the $X_{3}$ axis and its own axis. Then, eq 11 reduces to

$$
\begin{aligned}
\varepsilon_{33}^{\prime}= & \cos ^{2} \phi_{3} \varepsilon_{33}+(1 / 2)\left(1-\cos ^{2} \phi_{3}\right)\left(\varepsilon_{11}+\varepsilon_{22}\right) \\
= & \left\{\cos ^{2} \phi_{3} \varepsilon_{33}+(1 / 2)\left(1-\cos ^{2} \phi_{3}\right)\right. \\
& \left.\times\left(S_{13}+S_{23}\right)\right\} \sigma
\end{aligned}
$$

With the relationship $S_{33}^{\prime}=\varepsilon_{33}^{\prime} / \sigma$, eq 12 is given by

$$
\begin{aligned}
S_{33}^{\prime}= & \cos ^{2} \phi_{3} S_{33}+(1 / 2)\left(1-\cos ^{2} \phi_{3}\right)\left(S_{13}+S_{23}\right) \\
= & \left\{\cos ^{2} \phi_{3}+(1 / 2)\left(1-\cos ^{2} \phi_{3}\right)\right. \\
& \left.\times\left(S_{13}+S_{23}\right) / S_{33}\right\} S_{33}
\end{aligned}
$$

According to an estimate by Hibi et al., ${ }^{33}$ the compliances $S_{13}$ and $S_{23}$ can be represented as

$$
\begin{aligned}
& S_{13}=-v_{31} S_{33} \\
& S_{23}=-v_{32} S_{33}
\end{aligned}
$$

Thus, eq 13 can be rewitten as

$S_{33}^{\prime}=$

$\left\{\cos ^{2} \phi_{3}-(1 / 2)\left(1-\cos ^{2} \phi_{3}\right)\left(v_{31}+v_{32}\right)\right\} S_{33}$

The crystal lattice modulus $E_{\mathrm{c}}^{0}$ in the $c$-axis (or fiber axis) is represented by using the apparent 
modulus $E_{\mathrm{c}}$ of the (105) plane. That is,

$E_{\mathrm{c}}^{0}=\left\{\cos ^{2} \phi_{3}+(1 / 2)\left(1-\cos ^{2} \phi_{3}\right)\left(v_{31}+v_{32}\right)\right\} E_{\mathrm{c}}$

The angle $\phi_{3}$ was estimated to be $9.6^{\circ}$ in the geometrical arrangement of the X-ray diffraction measurement. Furthermore, if the $c$-axes are oriented in the $X_{3}$ axis perfectly, $\cos \phi_{3}$, from the crystallographic viewpoint, is given by

$$
\cos \phi_{3}=\frac{5 a b\left(1-\cos ^{2} \alpha-\cos ^{2} \beta-\cos ^{2} \gamma+2 \cos \alpha \cos \beta \cos \gamma\right)^{1 / 2}}{b^{2} c^{2} \sin ^{2} \alpha+25 a^{2} b^{2} \sin ^{2} \gamma-10 a b^{2} c(\cos \gamma \cos \alpha-\cos \beta)^{1 / 2}}
$$

where the values of all the coefficients in eq 17 were given by Daubeny et al. ${ }^{24}$ Substituting the values into eq 17 , we find $\phi_{3}=9.77$. This indicates that the $c$-axis are oriented predominantly in the $X_{3}$ axis and the mathematical evaluation by eq $9-16$ is valid.

Figure 10 shows the relationship between the crystal strain of the (105) plane and the external applied stress. The relationship can be represented as a straight line. The apparent modulus $E_{\mathrm{c}}$ of the (105) plane calculated from the slope of the straight line are almost in the range $115-126 \mathrm{GPa}$.

Figure 11 shows the relationship which can be realized by the superposition of the four figures in Figure 10. The apparent modulus $E_{\mathrm{c}}$ can be estimated to be $123 \mathrm{GPa}$. This value is a little bit higher than that reported by Sakurada et al. $(108 \mathrm{GPa}){ }^{32}$ This small difference is probably due to the differences of crystallinities, molecular orientations, and crystal sizes but the exact reason remains as unresolved problems.

Since the Poisson's ratio must be in the range $0-0.5$, we can estimate the crystal lattice modulus as follows:

$$
\begin{array}{ll}
v_{31}=v_{32}=0.5 & E_{\mathrm{c}}^{0}=118 \mathrm{GPa} \\
v_{31}=v_{32}=0.3 & E_{\mathrm{c}}^{0}=118 \mathrm{GPa} \\
v_{31}=v_{32}=0 & E_{\mathrm{c}}^{0}=119 \mathrm{GPa}
\end{array}
$$

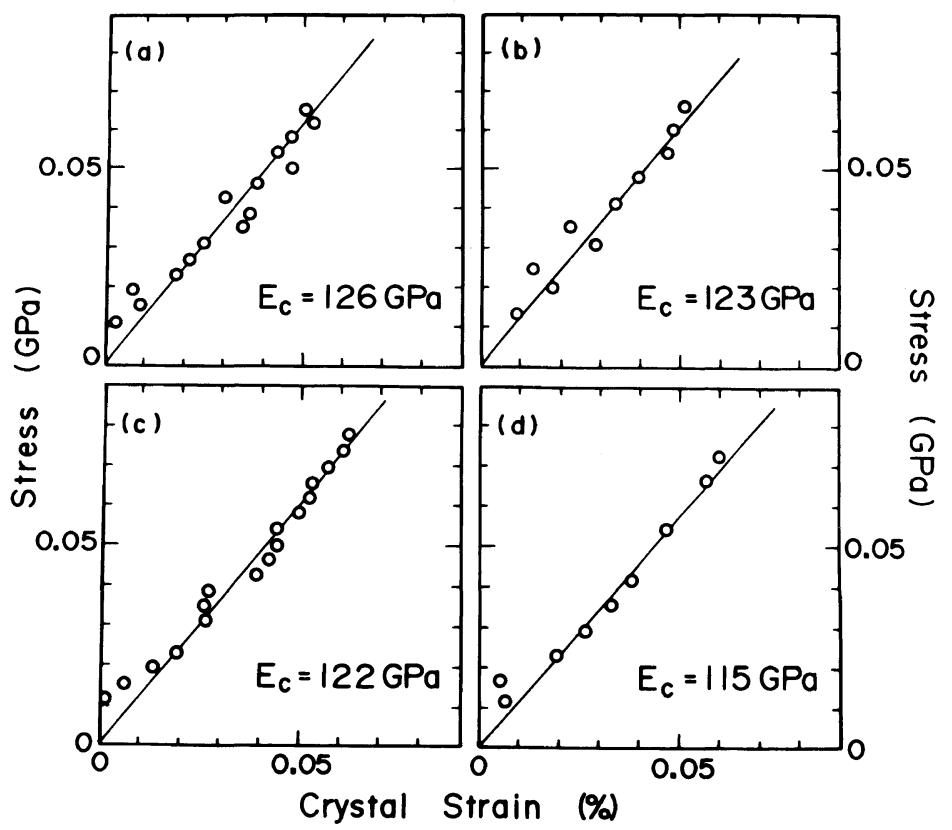

Figure 10. Stress-strain relationship for the (105) plane of PET films whose draw ratio is close to $\lambda=20$. 


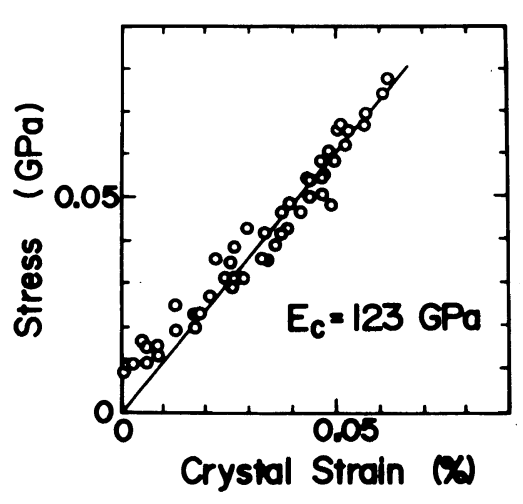

Figure 11. Stress-strain relationship for the (105) plane of PET films which can be realized by the superposition of the four relationships in Figure 10.

Thus it turns out that the crystal lattice modulus of PET is about $118 \mathrm{GPa}$. This value is in good agreement with the theoretical one $(95 \mathrm{GPa})$ reported by Tashiro et al. ${ }^{34}$ The rectification should be carried out using the geometrical arrangement concerning stress and strain relationship shown in Fig. 9 but the difference between the apparent crystal lattice modulus of the (105) plane and the real crystal modulus is not so significant.

Recently, the crystal lattice modulus was reported by Thistlethwaite et al. ${ }^{35}$ According to their report, ${ }^{35}$ the apparent modulus of the (105) plane was estimated in the range $65-83 \mathrm{GPa}$. These values are much lower than those measured by Sakurada et al. ${ }^{31}$ and in the present paper and are rather close to the previous work by Sakurada et al. ${ }^{36}$ They concluded that the real crystal modulus can be estimated to be $110 \pm 10 \mathrm{GPa}$ from the apparent values $65-83 \mathrm{GPa}$ by a rectification using a linear parallel-series model. Therefore, it is evident that the considerable increase in the real value after the rectification depends on a significant effect of the parallel-point on the model system. The modified values are also close to the present values $118-119 \mathrm{GPa}$. This indicates that the homegeneous stress hypothesis can be valid within the specimen used in this experiment.
Here it should be noted that the crystal lattice modulus is much higher than the strorage modulus $(19.5 \mathrm{GPa})$ of the drawn film with $\lambda=20$ at $20^{\circ} \mathrm{C}$. The second order orientation factor, 0.686 , of the $c$-axis is lower than the theoretical value, 0.974 , calculated at a draw ratio of 20 by assuming affine fashion. ${ }^{31}$ This indicates that the preferential orientation of the $c$-axis is not arisen by transmitting drawing force effectively. It would be expected that during the elongation in a hot water bath at $95^{\circ} \mathrm{C}$, the slippage of the amorphous chain segments is predominant and it does not cause any significant orientation of the segments.

In order to study the deformation mechanism of the PET films in detail, SAXS patterns were observed. Figure 12 shows the SAXS patterns from the dry gel films with $\lambda=1$ and 7. At $\lambda=1$, the pattern shows circular-type scattering and that at $\lambda=7$ shows meridional scattering having a scattering maxima corresponding to a long period of about $80 \AA$. The pattern in part (a) shows a random orientation of crystal lamellae and that in part (b) indicates that the drawn film is composed of crystal lamellae that are oriented predominantly with their large flat faces perpendicular to the stretching direction.

Figure 13 shows SAXS patterns from the drawn melt film with $\lambda=6$, in which the pattern in part (a) shows the scattering from the film drawn in a hot water bath at $65^{\circ} \mathrm{C}$ and the pattern in part (b) shows the scattering from the specimen annealed at $245^{\circ} \mathrm{C}$ for $20 \mathrm{~min}$ and cooled slowly to room temperature, after the drawing. Through the annealing, the crystallinity increased from 32 to $50 \%$ and the indistinct lobes of the scattering maximum in the pattern (a) extended in the horizontal direction become clearer and shorter, indicating increases of the perfectness of crystal lamellae and crystal lamellar length. In contrast, the long period is hardly affected by the annealing and remains $80 \AA ̊$.

Figure 14 shows SAXS patterns of the undrawn and drawn films. The pattern in part 
Gel Film

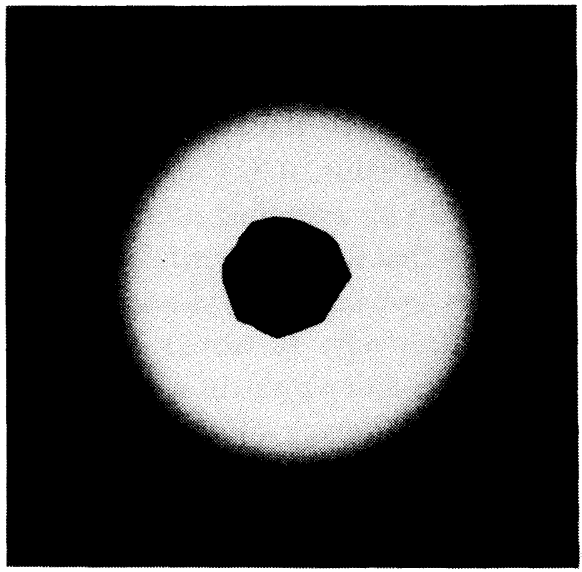

(a) $\lambda=1$

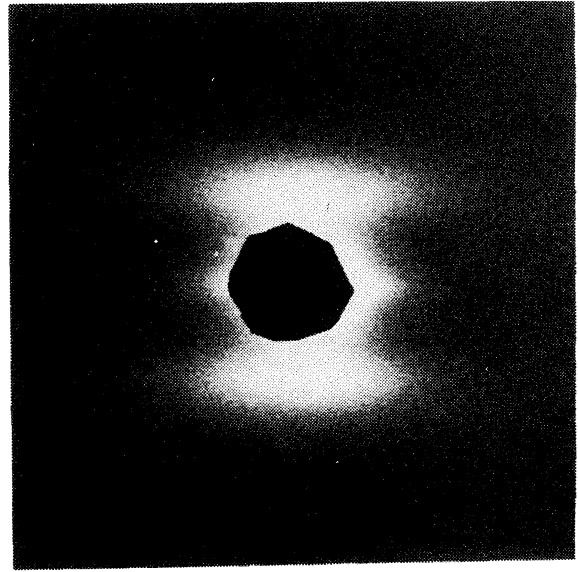

(b) $\lambda=7$

Figure 12. SAXS patterns from PET gel films. (a) $\lambda=1$; (b) $\lambda=7$.

Melt Pressed Film

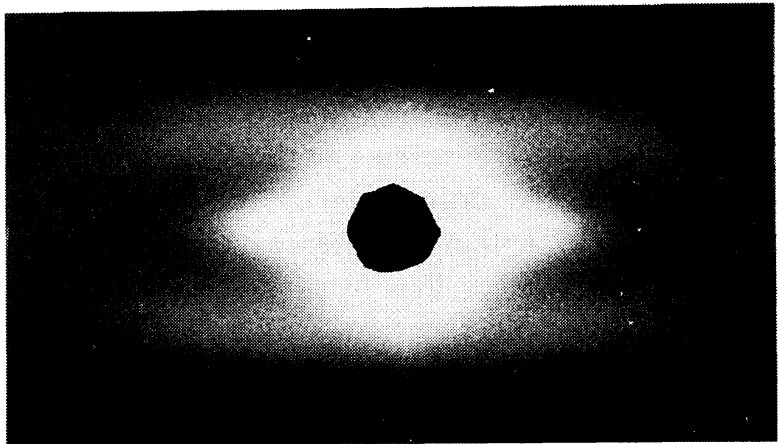

(a)

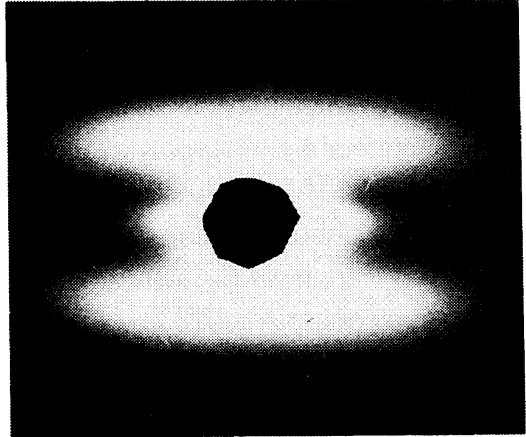

(b)

Figure 13. SAXS patterns from PET melt films with a drawn ratio of 6: pattern (a) from the film drawn in a hot water bath at $65^{\circ} \mathrm{C}$ and pattern (b) from the film annealed at $245^{\circ} \mathrm{C}$ for $20 \mathrm{~min}$ and cooled down to room temperature slowly after the drawing in the indicated condition.

(a) of the undrawn film shows circular diffuse scattering and the pattern in part (b) of the film drawn to $\lambda=12$ in a hot water bath at $95^{\circ} \mathrm{C}$, shows a diffuse scattering maximum corresponding to a long period of about $60 \AA$. This indicates that the existence of the crystal lamellae is due to the oriented crystallization by the elongation in a hot water at $95^{\circ} \mathrm{C}$. The diffuse scattering lobes in the pattern (b) become clear through the annealing at $245^{\circ} \mathrm{C}$ for $20 \mathrm{~min}$, as shown in the pattern (c). The long period, however, is independent of the annealing and the value remains as $63 \AA$ in spite of an increase in crystallinity from 29 to $52 \%$. The pattern in part (d) shows the scattering from the film which was elongated to $\lambda=20$ in a hot oven at $245^{\circ} \mathrm{C}$ after drawing to $\lambda=12$ in a hot water bath at $95^{\circ} \mathrm{C}$. The scattering maximum shifts to the center and the long period becomes $80 \AA$ with increasing crystallin- 
Melt Pressed Film

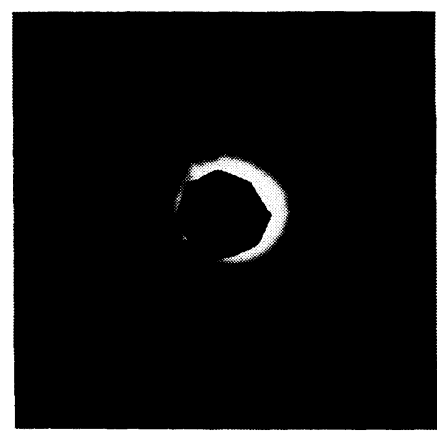

(a) $\lambda=1$

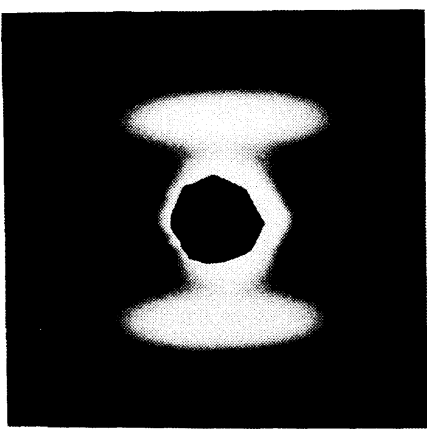

(c) $\lambda=12$

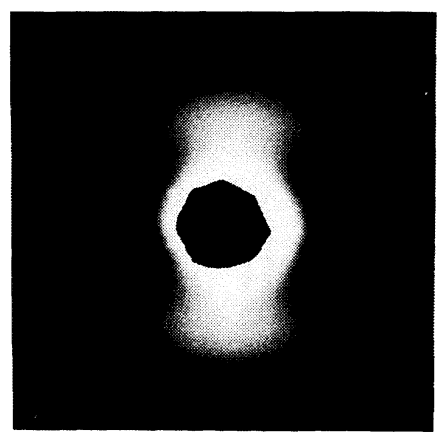

(b) $\lambda=12$

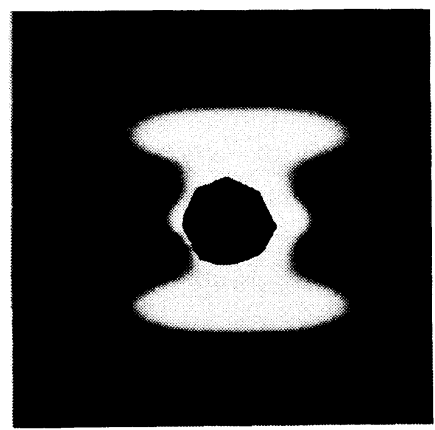

(d) $\lambda=20$

Figure 14. SAXS patterns from melt films of PET: pattern (a) from the undrawn film, pattern (b) from the drawn film to $\lambda=12$ in a hot water bath at $95^{\circ} \mathrm{C}$, pattern (c) from the annealed film at $245^{\circ} \mathrm{C}$ for $20 \mathrm{~min}$ and cooled down slowly to room temperature after the drawing, pattern (d) from the film drawn from $\lambda=12$ to 20 at $245^{\circ} \mathrm{C}$.

ity from 52 to $60 \%$. Even for this specimen, the long period is less than $100 \AA$. In contrast, long periods of ultradrawn polyethylene films are expected to be above $300 \AA$, since they could not be detected by our X-ray instrument whose maximum length of a optical bench is about $40 \mathrm{~cm}$. This means that the preferential orientation of the $c$-axes of the PET film with respect to the stretching direction by the elongation in a hot oven at $245^{\circ} \mathrm{C}$ is mainly due to the rotation of crystallites leading to taut tie molecules but not to a significant transition from a folded to a fibrous type like ultradrawn polyethylene films. This orientation of crystallites hampers the ultradrawing of PET film.

In order to facilitate understanding of the above concept, we refer to a quantitative evaluation of the change in the SAXS patterns by drawing and annealing processes discussed before. In attempting theoretical calculations of SAXS patterns, we propose a twodimensional model system in order to derive an expression for the distribution of intensity in the SAXS pattern for a system of stacked folded-chain lamellae in which there are orientational fluctuation. In the theory, a realistic mathematical treatment is made, based on the concept that the scattering is caused by positive and negative derivations from the average density of the sample, ${ }^{38,39}$ since the scattering is attributed to an alternation in texture between crystalline and amorphous intercrystalline regions where both regions 


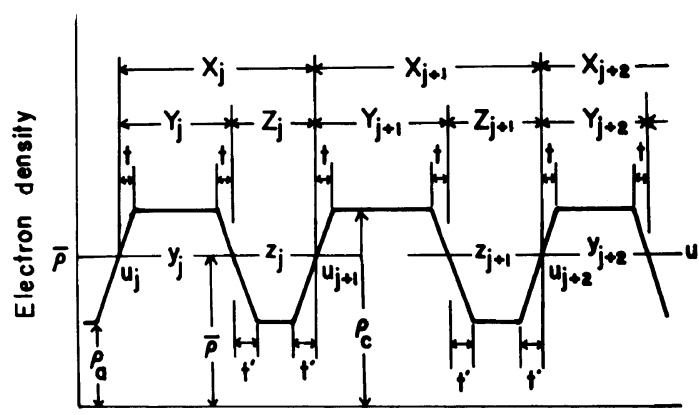

Figure 15. The model system used for the theoretical analysis of the SAXS intensity distribution.

fluctuate in thickness about their respective mean value.

The coordinate system is given in the case where a film is composed of large lamellar crystals stacked on top of one another to form a periodic crystalline-amorphous structure perpendicular to the stretching direction. Figure 15 shows the coordinate, in which the $X_{3}$ corresponds to the stretching direction and the $X_{1}$ axis is parallel to the direction of an incident beam whose unit vector is defined as $s_{0}$. The intensity distribution is observed as a function of the scattering angle $2 \theta$ and the azimuthal angle $\mu$ and $s^{\prime}$ is a unit vector along the scattering X-ray beam. In order to simplify the calculation, it is assumed that the crystalline lamellae are rectangular in cross-section and situated in the plane $0-X_{2} X_{3}$. The center of gravity of each crystalline lamellae is in the direction of the $u$ axis. The dimensions of the $j$-th lamellae are on the $0-X_{2} X_{3}$ plane. The size of lamellae in the $X_{1}$ direction is neglected, since this affects only on the magnitude of the scattered intensity but is independent of the intensity distribution. In this model system, it is assumed that all the lamellae have same length $\bar{L}$. The distance between the $j$-th and the $(j+1)$-th lamellae is defined as $Z_{j}$ and $\alpha$ denotes the orientational angle between $X_{3}$ and $u$ axes.

In order to represent the variation of density along the $u$ axis, a schematic diagram in Figure 16 is proposed, in which the variation is specified by a trapezoidal function. Inciden-

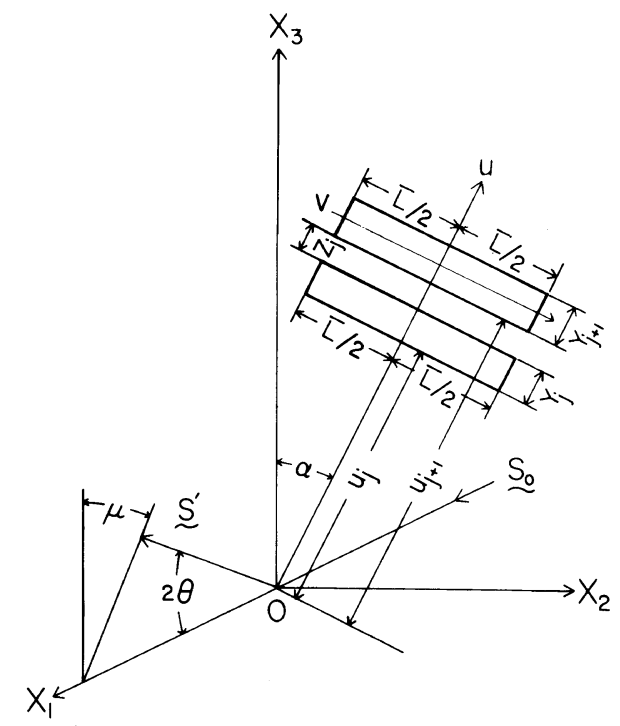

Figure 16. The model system for representing the SAXS intensity distribution, in which the density distribution between the two phases is specified by positive and negative derivations from the average of the system.

tally, the density variation along the $v$ axis in Figure 15 is neglected. The lengths $Y_{j}$ and $Z_{j}$ are those of the representive phases and $X_{j}$ is the total length (the repeat distance) of the $j$-th pair. The lengths $t$ and $t^{\prime}$ of the transition zones are assumed to be constant whereas the lengths $Y_{j}$ and $Z_{j}$ are assumed to fluctuate independently from the average values $\bar{Y}$ and $\bar{Z}$.

According to the previous work, ${ }^{39}$ the scattered intensity is given by

$$
\frac{1}{\bar{N} \bar{L} \bar{X}}=\frac{1}{\bar{N} \bar{L} \bar{X}}\left(I_{\mathrm{B}}-I_{\mathrm{C}}\right) / \int_{0}^{2 \pi} p(\alpha) \mathrm{d} \alpha
$$

where $\bar{X}(=\bar{Y}+\bar{Z})$ and $\bar{N}$ denote the mean periodic distance and the average number of crystal lamellae, respectively. $p(\alpha)$ in eq 19 is the orientation distribution function of the lamellar assembly with respect to the scattering direction. The derivations of the $I_{\mathrm{B}}$ and $I_{\mathrm{C}}$ terms are described in the Appendix.

Figures 17-19 show the aximuthal angle $(\mu)$-dependence of the scattered intensity distribution calculated with various values of $\bar{X} / \bar{L}, t$, and $p$. In carrying out the calculations, 


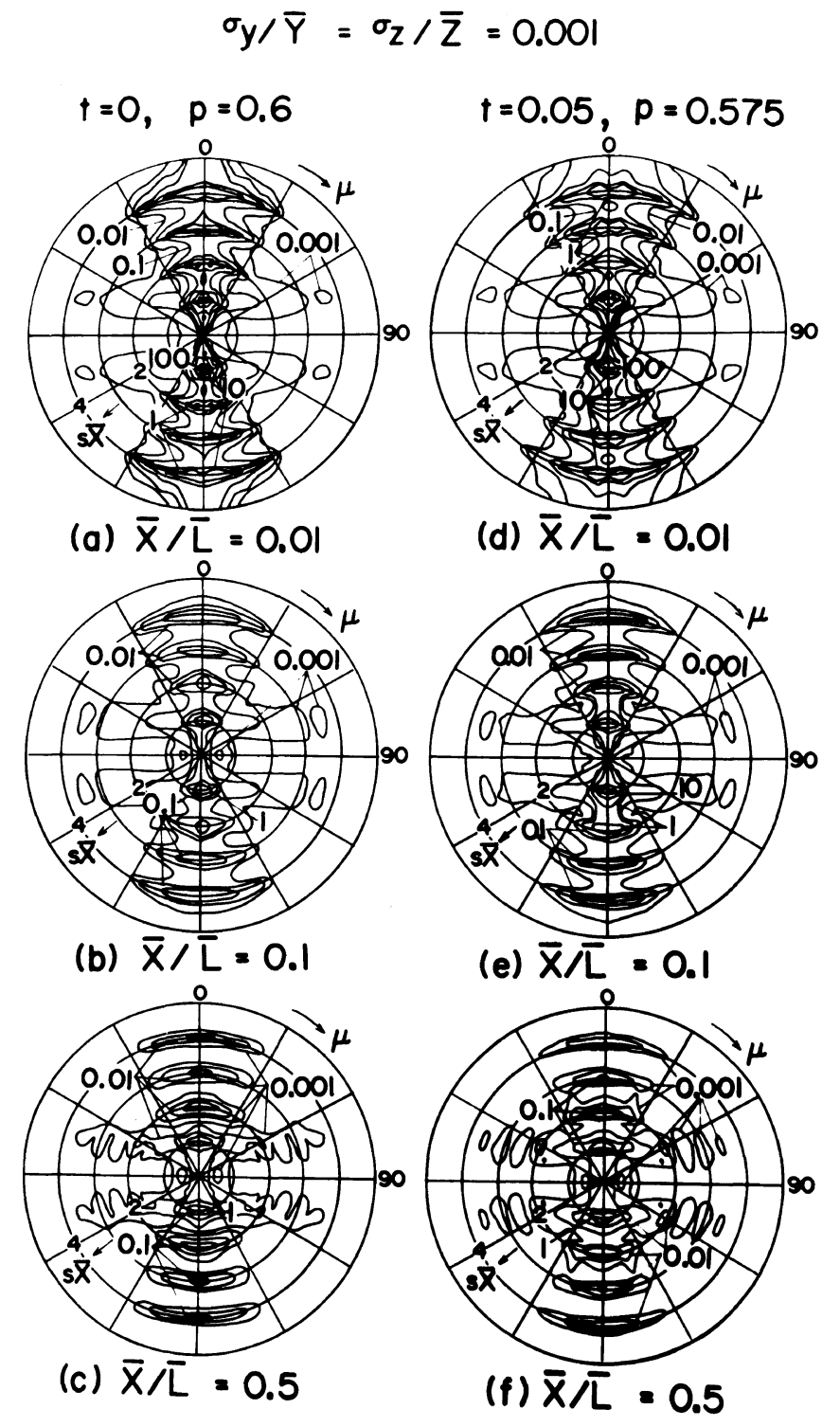

Figure 17. SAXS patterns calculated with the variable indicated parameters at fixed state of $\sigma_{y} / \bar{Y}=\sigma_{z} / \bar{Z}=0.001$.

we assume that the parameters describing the fluctuation of the periodic distance were set at very small values such as $\sigma_{y} / \bar{Y}=\sigma_{z} / \bar{Z}=0.001$. The patterns on the left side are calculated by neglecting the effect of the transition zone $t$, while those on the right side are calculated by introducing the effect of $t$. When $t=0, p$ corresponds to $X_{c}$ and is given as 0.6 . In contrast, when $t / \bar{X}=0.05, p$ must be 0.575 to remain as $X_{\mathrm{c}}=0.6$. The profiles of the patterns are sensitive to the changes in $\bar{X} / \bar{L}$. The meridional scattering maxima become distinct at $s \bar{X} \geqq 2$. For $\bar{X} / \bar{L} \leqq 0.1$, the profiles of the patterns on both sides are of similar shape and this indicates that the introduction of the transition zone is not effective enough to 


$$
\sigma_{\mathrm{y}} / \overline{\mathrm{Y}}=\sigma_{\mathrm{z}} / \overline{\mathrm{Z}}=0.1
$$
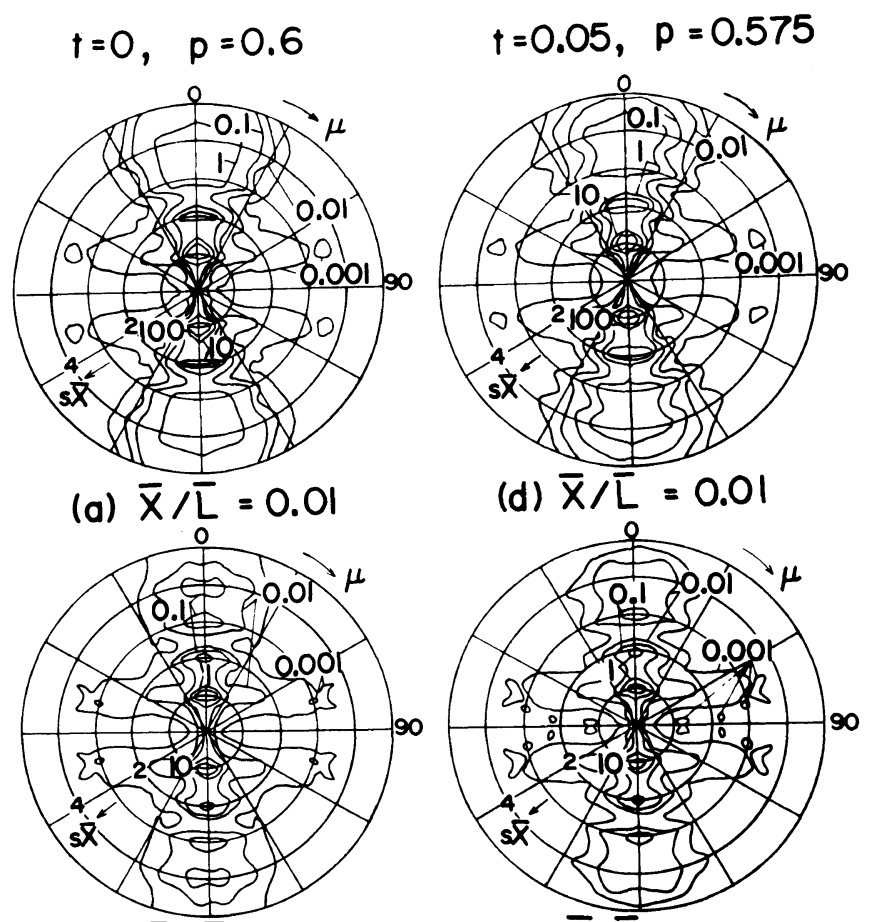

(b) $\bar{X} / \bar{L}=0.1$
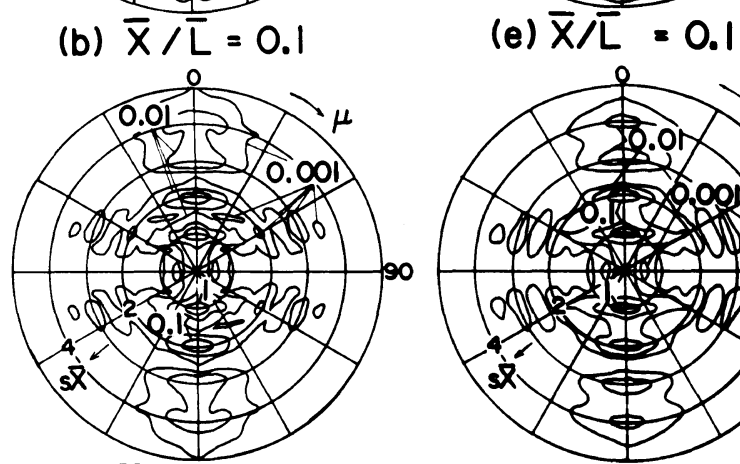

(c) $\bar{X} / \bar{L}=0.5$

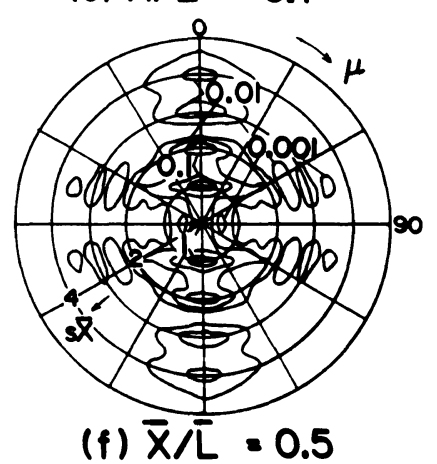

Figure 18. SAXS patterns calculated with the variable indicated parameters at fixed state of $\sigma_{y} / \bar{Y}=\sigma_{z} / \bar{Z}=0.1$.

change the profile. At $\bar{X} / \bar{L}=0.5$, however, the scattering lobes are derived into the many scattering lobes in the horizontal direction.

Figure 18 shows the $(\mu)$-dependence of the scattered intensity for various values of $\bar{X} / \bar{L}$, $t$, and $p$. In calculations, the parameters $\sigma_{y} / \bar{Y}$, and $\sigma_{z} / \bar{Z}$ were set to be 0.1 . In this system, the fluctuation of the periodic distance is larger than that in Figure 17. Comparing the patterns on the left side with those on the right side, the effect of the transition zone does not cause any significant change in the profiles. The relative increase in the periodic distance against the lamellar length makes the shapes of the lobes concerning the first and second order scattering maxima more complicated. The 


$$
\sigma_{\mathrm{y}} / \overline{\mathrm{Y}}=\sigma_{\mathrm{z}} / \overline{\mathrm{Z}}=0.5
$$
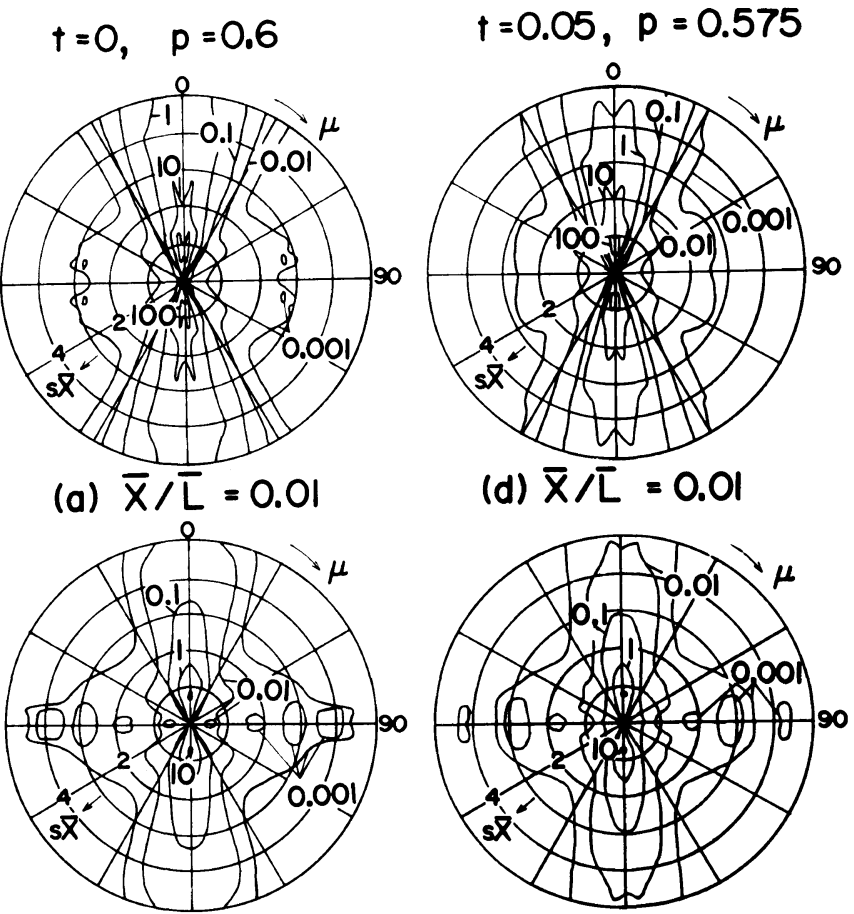

(b) $\bar{X} / \bar{L}=0.1$
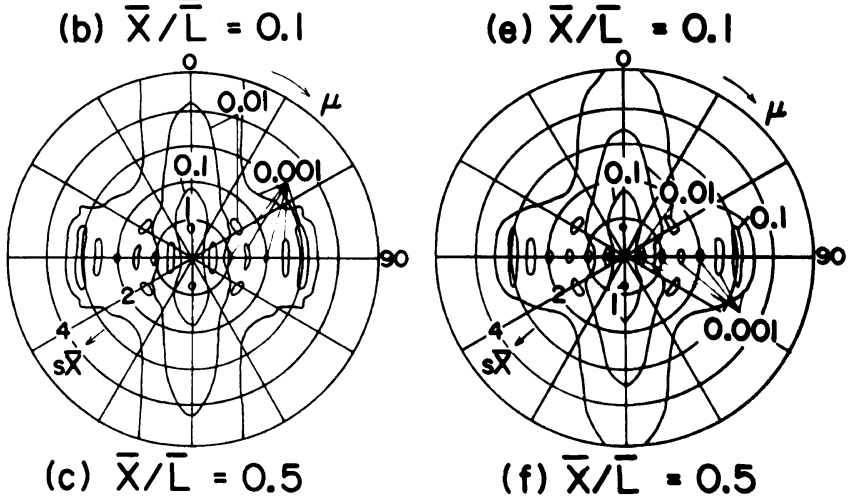

Figure 19. SAXS patterns calculated with the variable indicated parameters at fixed state of $\sigma_{y} / \bar{Y}=\sigma_{z} / \bar{Z}=0.5$.

comparison between the patterns in Figures 17 and 18 indicates that the scattering maxima become unclearer as the fluctuation of the periodic distance increases.

The further increases in $\sigma_{y} / \bar{Y}$ and $\sigma_{z} / \bar{Z}$ cause the disappearance of the scattering maxima in the meridional direction as shown in Figure 19 and the intensity mimima like a valley appeared in the horizontal direction. The intensity distribution in the meridional direction shows a monotonious decreasing curve and a degree of the decrease becomes duller with increasing $\bar{X} / \bar{L}$.

Judging from these patterns in Figures 17-18, an increase in the lamellar length $\bar{L}$ against the periodic distance $\bar{X}$ is thought to 
cause a decrease in the scattered intensity in the meridional direction and this tendency becomes marked for the first order maximum. As discussed before, it is evident that the profiles of the patterns are sensitive to the changes in $\bar{X} / \bar{L}$. Accordingly, it may be concluded that the change in the patterns in Figures $12-14$ by the annealing can be accounted for in terms of the changes in $\bar{X} / \bar{L}$ in addition to the changes of $\sigma_{y} / \bar{Y}$ and $\sigma_{z} / \bar{Z}$.

Here it can be seen that the profiles of the patterns in Figures 17-19 become more distinct with decreasing $\bar{X} / \bar{L}, \sigma_{y} / \bar{Y}$, and $\sigma_{z} / \bar{Z}$. This means that the change in the profiles by the annealing as shown in Figure 13 is probably due to an increase in the lamellar length as well as a decrease in the fluctuation of the periodic distance. Furthermore the change from the pattern (b) to the pattern (c) in Figure 14 by the annealing may be explained as a decrease in the fluctuation of the periodic distance with the development of lamellar tissue, since the scattering lobes in the meridional direction becomes distinct by decreasing the values of $\sigma_{y} / \bar{Y}$ and $\sigma_{z} / \bar{Z}$, as shown in Figures 17 and 18 . This indicates the effect of lamellar growth by the annealing.

Through a series of experiments, it may be concluded that the poor drawability of PET gel melt films is attributed to the orientation of crystallites; the preferential orientation of the $c$-axes is only due to the rotation of crystallites leading to taut tie molecules but not to a significant transformation from a folded to a fibrous type, since there exist lamellae whose long periods are less than $100 \AA$ and the orientational degree of the $c$-axis is not as great as that of the amorphous chain segments.

\section{CONCLUSIONS}

PET gel and melt films were elongated up to $\lambda=7$ and 20 , respectively, corresponding to their maximum draw ratios. The orientation distribution of crystallites was investigaged in terms of the second order orientation factors of three principal crystallographic axes calculated by using the orientation factors of the reciprocal lattice vectors of the (100), (110), $(010),(1 \overline{1} 1)$, and $(0 \overline{1} 1)$ planes. The values of the orientation factors of three principal crystallographic axes indicate that the orientation of crystallites within the gel and melt films are of similar shapes of an ideal fiber. The storage moduli of the gel and melt films are 16.2 and $19.5 \mathrm{GPa}$, respectively, at $20^{\circ} \mathrm{C}$. In order to obtain the ultimate value of the Young's modulus of PET, the crystal lattice modulus of the $c$-axis was measured by the $\mathrm{X}$-ray diffraction and the value was estimated to be $118 \mathrm{GPa}$. This indicates that the storage moduli for both the specimens are much lower than the crystal lattice modulus. This large difference between the storage modulus and the crystal lattice modulus was attributed to the preferential orientation mechanism of the $c$-axes due to the rotation of crystallites leading to taut tie molecules but not to a crystal transformation from a folded to a fibrous type. This mechanism hampers ultradrawing of PET to assure high modulus and high strength. The existence of crystal lamellae with periodic distances $<100 \AA$ also supports the crystal rotation to realize the preferential orientation of the $c$-axes with respect to the stretching direction, without the crystal transformation.

\section{APPENDIX}

Following the previous paper, ${ }^{39}$ the $I_{\mathrm{B}}$ and $I_{\mathrm{C}}$ terms are given by

$$
I_{\mathrm{B}}=I_{1}^{\mathrm{B}}+I_{2}^{\mathrm{B}}+I_{3}^{\mathrm{B}}
$$

and

$$
I_{\mathrm{C}}=I_{1}^{\mathrm{C}}+I_{2}^{\mathrm{C}}+I_{3}^{\mathrm{C}}
$$

where

$$
I_{1}^{\mathrm{B}}=\int_{0}^{2 \pi} p(\alpha) \operatorname{Re}\left\{\frac{1}{\bar{X} \bar{L}}\left[J_{1}+\frac{2 G_{y} \gamma_{y} F_{z}}{1-F_{y} F_{z}}\right]\right\} \mathrm{d} \alpha
$$




$$
\begin{aligned}
I_{2}^{\mathrm{B}}= & \int_{0}^{2 \pi} p(\alpha) \operatorname{Re}\left\{\frac{1}{\bar{X} \bar{L}}\left[J_{2}+\frac{2 A_{z} B_{z} F_{y}}{1-F_{y} F_{z}}\right]\right\} \mathrm{d} \alpha \\
I_{3}^{\mathrm{B}}= & \left.\int_{0}^{2 \pi} p(\alpha) \operatorname{Re}\left\{\frac{1}{\bar{X} \bar{L}}\left[\frac{2\left(G_{y} B_{z}+A_{z} \gamma_{y}\right)}{1-F_{y} F_{z}}\right]\right\} \mathrm{A}-2-\mathrm{b}\right) \\
I_{1}^{\mathrm{C}}= & \int_{0}^{2 \pi} p(\alpha) \operatorname{Re}\left\{\frac{2 G_{y} \gamma_{y} F_{z}\left[1-\left(F_{y} F_{z}\right)^{N}\right]}{N \bar{X} \bar{L}\left(1-F_{y} F_{z}\right)^{2}}\right\} \mathrm{d} \alpha \\
I_{2}^{\mathrm{C}}= & \int_{0}^{2 \pi} p(\alpha) \operatorname{Re}\left\{\frac{2 A_{z} B_{z} F_{y}\left[1-\left(F_{y} F_{z}\right)^{N}\right]}{N \bar{X} \bar{L}\left(1-F_{y} F_{z}\right)^{2}}\right\} \mathrm{d} \alpha \\
I_{3}^{\mathrm{C}}= & \left.\int_{0}^{2 \pi} p(\alpha)-\mathrm{d}-2-\mathrm{e}\right) \\
& \times \operatorname{Re}\left\{\frac{2\left(G_{y} B_{z} F_{y} F_{z}+\gamma_{y} A_{z}\right)\left[1-\left(F_{y} F_{z}\right)^{N}\right]}{N \bar{X} \bar{L}\left(1-F_{y} F_{z}\right)^{2}}\right\} \mathrm{d} \alpha
\end{aligned}
$$

where

$$
\begin{aligned}
& J_{1}=\int_{-\infty}^{\infty}\left|f_{j}\right|^{2} H\left(Y_{j}\right) \mathrm{d} Y_{j} \\
& J_{2}=\int_{-\infty}^{\infty}\left|g_{j}\right|^{2} h\left(Z_{j}\right) \mathrm{d} Z_{j} \\
& G_{y}=\int_{-\infty}^{\infty} f_{j}^{*} H\left(Y_{j}\right) \exp \left(-2 \pi i b Y_{j}\right) \mathrm{d} Y_{j} \\
& F_{y}=\int_{-\infty}^{\infty} H\left(Y_{j}\right) \exp \left(-2 \pi i b Y_{j}\right) \mathrm{d} Y_{j} \\
& \gamma_{y}=\int_{-\infty}^{\infty} f_{j} H\left(Y_{j}\right) \mathrm{d} Y_{j} \\
& A_{z}=\int_{-\infty}^{\infty} g_{j}^{*} h\left(Z_{j}\right) \exp \left(-2 \pi i b Z_{j}\right) \mathrm{d} Z_{j} \\
& F_{z}=\int_{-\infty}^{\infty} h\left(Z_{j}\right) \exp \left(-2 \pi i b Z_{j}\right) \mathrm{d} Z_{j} \\
& B_{z}=\int_{-\infty}^{\infty} g_{j} h\left(Z_{j}\right) \mathrm{d} Z_{j}
\end{aligned}
$$

Following Blundell, ${ }^{40}$ we assume that the variations of the lengths $Y_{j}$ and $Z_{j}$ are given by symmetrical Gaussian functions with the respective mean lengths of $\bar{Y}$ and $\bar{Z}$, and the standard derivations $\sigma_{y}$ and $\sigma_{z}$; thus

$$
H\left(Y_{j}\right)=(2 \pi)^{-1 / 2} \sigma_{y}^{-1} \exp \left[-\left(Y_{j}-\bar{Y}\right)^{2} / 2 \sigma_{y}^{2}\right]
$$

$$
h\left(Z_{j}\right)=(2 \pi)^{-1 / 2} \sigma_{z}^{-1} \exp \left[-\left(Z_{j}-\bar{Z}\right)^{2} / 2 \sigma_{z}^{2}\right]
$$

Using the trapexium model in Figure 16, the terms $I_{\mathrm{B}}$ and $I_{\mathrm{C}}$ can be formulated as concrete forms and they were described elsewhere. ${ }^{39}$

Considering a real system, the portion of the specimen sampled by the X-ray beam contains a distribution of $N$ lamellae. This concept must be introduced in order to smear out many subsidiary maxima that appear at lower scattering angles $s \bar{X}$. This has been taken into consideration by Hashimoto et al. ${ }^{41}$ by introducing a discrete symmetrical distribution of $N$ with standard drivation $\sigma_{N}=3$. This distribution is written as

$P(N)=$

$\exp \left\{-\frac{(N-\bar{N})^{2}}{2 \sigma_{N}^{2}}\right\} / \sum_{N=1}^{2 \bar{N}-1} \exp \left\{-\frac{(N-\bar{N})^{2}}{2 \sigma_{N}^{2}}\right\}$

In the present work, we will assume the same type of distribution of $N$. Then the average value of the term $I_{C}$ is given by

$$
\left\langle\mathrm{I}_{\mathrm{C}}\right\rangle_{\mathrm{av}}=\sum_{N=1}^{2 \bar{N}-1} I_{\mathrm{C}} P(N)
$$

In our subsequent calculations, we assume an average $\bar{N}$ of 10 .

The expressions for the SAXS intensity distribution contain five parameters, $\sigma_{y} / \bar{Y}$, $\sigma_{z} / \bar{Z}, \bar{X} / \bar{L}, p(=\bar{Y} / \bar{X})$, and volume crystallinity $X_{c}$, as described elsewhere, ${ }^{39}$ and the values of these parameters must be determined on carrying out numerical calculations. Incidentally, the parameters $t$ and $t^{\prime}$ denoting the transition zone may be given by using $p$ and $x_{\mathrm{c}}$, as follows

$$
\frac{t}{\bar{X}}=\frac{\left(1-X_{\mathrm{c}}\right)\left(p-X_{\mathrm{c}}\right)}{1-2 X_{\mathrm{c}}}
$$




$$
\frac{t^{\prime}}{\bar{X}}=\frac{X_{\mathrm{c}}\left(p-X_{\mathrm{c}}\right)}{1-2 X_{\mathrm{c}}}
$$

The crystallinity $X_{c}$ can be readily estimated by a density measurement but $p$ cannot be determined. In a real system, however, $t$ and $t^{\prime}$ are usually much smaller than $\bar{Y}$ and thus for numerical calculations, $p$ can be assumed to be essentially close to $X_{\mathrm{c}}$.

To complete theoretical analysis, the orientation distribution function of crystal lamellae $p(\alpha)$ is defined by

$$
p(\alpha)=\exp \left[-\sigma_{\alpha}^{2} \sin ^{2} \alpha\right]
$$

where $\sigma_{\alpha}$ is a parameter associated with the shape of $p(\alpha)$. When $\sigma_{\alpha}$ is a large value, $p(\alpha)$ shows a sharp distribution. In contrast, when $\sigma_{\alpha}=0, p(\alpha)$ shows random distribution. In our subsequent calculations, $\sigma_{\alpha}$ is assumed to be 5 , since the theoretical patterns calculated at $\sigma_{\alpha}=5$ were found to give the best fit to the observed patterns in Figures 13-15 through trial and error.

\section{REFERENCES}

1. J. Smook, J. C. Torf, P. F. van Hutlten, and A. J. Pennings, Polym. Bull., 2, 293 (1980).

2. P. J. Barham and A. Keller, J. Mater. Sci., 15, 2229 (1980).

3. A. Zwijnenburg and A. J. Pennings, Coll. Polym. Sci., 253, 452 (1975).

4. G. Cappaccio and I. M. Ward, J. Macromol. Sci.-Phys., B19(1), 35 (1974).

5. T. Kanamoto, E. S. Sherman, and R. S. Porter, Polym. J., 11, 497 (1979).

6. P. Smith and P. J. Lemstra, J. Mater. Sci., 15, 505 (1980).

7. P. Smith, P. J. Lemstra, and H. C. Booij, J. Polym. Sci., Polym. Phys. Ed., 19, 877 (1981).

8. C. Sawatari and M. Matsuo, Colloid. Polym. Sci., 263, 783 (1985).

9. A. Peney and R. S. T. Manley, Polym. Commun., 25, 39 (1984).

10. T. Kanamoto, A. Tsuruta, K. Tanaka, and M. Takeda, Polym. J., 16, 75 (1984).

11. M. Matsuo and C. Sawatari, Macromolecules, 19, 2036 (1986).

12. C. Sawatari and M. Matsuo, Macromolecules, 19, 2653 (1986)

13. N. Yoshihara, A. Fukushïma, Y. Watanabe, A.
Nakai, S. Nomura, and H. Kawai, Sen-i-Gakkaishi, 37, 387 (1981).

14. A. Misra and R. S. Stein, J. Polym. Sci., Polym. Phys. Ed., 17, 235 (1979).

15. J. Shimizu, N. Okui, and T. Kikutani, Sen-iGakkaishi, 34, 135 (1981).

16. J. Shimizu, N. Okui, and T. Kikutani, Sen-iGakkaishi, 37, 1981 (1981).

17. P. N. Peszkin, J. M. Shultz, and J. S. Lin, J. Polym. Sci., Polym. Phys. Ed., 24, 2591 (1986).

18. M. Ito, K. Tanaka, and T. Kanamoto, J. Polym. Sci., Polym. Phys., B, 25, 2127 (1987).

19. M. Matsuo, M. Tamada, T. Terada, C. Sawatari, and M. Niwa, Macromolecules, 15, 988 (1982).

20. T. Terada, C. Sawatari, T. Chigono, and M. Matsuo, Macromolecules, 15, 998 (1982).

21. R. Huisman and H. M. Huevel, J. Polym. Sci., Polym. Phys. Ed., 22, 943 (1978).

22. H. M. Heuvel and R. J. Huisman, J. Polym. Sci., Polym. Phys. Ed., 22, 2229 (1978).

23. C. Sawatari, T. Okumura, and M. Matsuo, Polym. $J .$, 18, 741 (1986).

24. R. deDaubeny, C. W. Bunn, and C. J. Brown, Proc. R. Soc. London, Ser. A, 226, 531 (1954).

25. M. Kashiwagi, A. Cunningham, A. J. Manuel, and I. M. Ward, Polymer, 15, 743 (1974).

26. J. H. Nobbs, D. I. Bower, I. M. Ward, and D. Patterson, Polymer, 15, 287 (1974).

27. A. Cunninghan, G. R. Davies, and I. M. Ward, Polymer, 15, 743 (1974).

28. R. J. Roe and W. R. Krigbaum, J. Chem. Phys., 40, 2608 (1964).

29. W. R. Krigbaum and Y. I. Balta, J. Phys. Chem., 71, 1770 (1967).

30. N. Sakaguchi, T. Oda, A. Nakai, and H. Kawai, Sen-i-Gakkaishi, 33, 499 (1977).

31. C. W. Bunn and R. DeDaubeny, Trans. Faraday Soc., 50, 1173 (1956).

32. I. Sakurada and K. Kaji, J. Polym. Sci., C, 31, 57 (1970).

33. S. Hibi, M. Maeda, M. Mizuno, S. Nomura, and H. Kawai, Sen-i-Gakkaishi, 29, 137 (1973).

34. K. Tashiro, M. Kobayashi, and H. Tadokoro, Macromolecules, 10, 413 (1977).

35. Y. Thistlethwaite, R. Jakeways, and I. M. Ward, Polymer, 29, 61 (1988).

36. I. Sakurada, T. Ito, K. Nakamae, J. Polym. Sci., C, 15, 75 (1966).

37. C. Sawatari and M. Matsuo, Macromolecules, 19, 2726 (1986)

38. M. Matsuo, C. Sawatari, M. Tsuji, R. S. T. Manley, J. Chem. Soc., Faraday Trans 2, 79, 1953 (1983).

39. M. Matsuo and C. Kitayama, Polym. J., 17, 4789 (1985).

40. D. J. Blundel, Acta Crystallogr. A26, 472 (1970).

41. T. Hashimoto, K. Nagatoshi, A. Todo, H. Hasegawa, and H. Kawai, Macromolecules, 7, 264 (1974). 